\title{
Cosmic microwave background bispectrum and inflation
}

\author{
Limin Wang* and Marc Kamionkowski ${ }^{\dagger}$ \\ Department of Physics, 538 West 120th Street, Columbia University, New York, New York 10027
}

(Received 5 August 1999; published 22 February 2000)

\begin{abstract}
We derive an expression for the non-Gaussian cosmic microwave background (CMB) statistic $I_{l}^{3}$ defined recently by Ferreira, Magueijo, and Górski in terms of the slow-roll-inflation parameters $\epsilon$ and $\eta$. This result shows that a nonzero value of $I_{l}^{3}$ in COBE would rule out single-field slow-roll inflation. A sharp change in the slope of the inflaton potential could increase the predicted value of $I_{l}^{3}$, but not significantly. This further suggests that it will be difficult to account for such a detection in multiple-field models in which density perturbations are produced by quantum fluctuations in the scalar field driving inflation. An Appendix shows how to evaluate an integral that is needed in our calculation as well as in more general calculations of CMB bispectra.

PACS number(s): 98.80.Cq, 98.80.Es
\end{abstract}

\section{INTRODUCTION}

Ferreira, Magueijo, and Górski (FMG) [1] have recently found evidence for a non-Gaussian distribution of cosmic microwave background (CMB) temperature fluctuations in the Cosmic Background Explorer (COBE) data (as have several other groups [2,3]). The common lore is that this result is inconsistent with the nearly Gaussian distribution of temperature fluctuations expected from inflation. Although the result may be due to foregrounds or some curious systematic effect [4-8], it is still worthwhile to state more precisely the implications for inflationary models if the non-Gaussianity is indeed in the CMB. That is the purpose of this paper. ${ }^{1}$

Inflation predicts the distribution of primordial perturbations to be very nearly Gaussian, but self-interactions of the inflaton field should in fact produce at least tiny deviations from Gaussianity [10-14]. We derive here an analytic expression for FMG's non-Gaussian statistic $I_{l}^{3}$ given in terms of the usual slow-roll parameters $\epsilon$ and $\eta$ in single-field slow-roll inflation models. Our results verify the common expectation that the detected value of $I_{l}^{3}$ is too large (by at least five orders of magnitude) to be consistent with slow-roll inflation. Motivated by evidence for a break in the power spectrum of the galaxy distribution [15-17], it is natural to consider inflation models in which the slope of the inflaton potential has a discontinuity $[18,19]$. We show that the increase of the predicted non-Gaussian signal is insignificant. We infer from this that such a large value of $I_{l}^{3}$ should also be hard to come by in multiple-field models in which density perturbations are produced by quantum fluctuations in the field driving inflation. (Counter-examples include the models discussed in Refs. [20-23].)

We begin by reviewing in Sec. II the calculation of the large-angle CMB power spectrum. We then move on in Sec. III to the calculation of the CMB bispectrum and present our result for $I_{l}^{3}$ in Sec. IV. Section V considers an inflation

\footnotetext{
*Email address: limin@astro.columbia.edu

†Email address: kamion@phys.columbia.edu

${ }^{1}$ For a review of the CMB and inflation, see [9].
}

model with a discontinuity in the slope of the inflaton potential. Section VI provides a discussion. An Appendix provides a recursive technique for evaluating an integral involving the product of three spherical Bessel functions that is needed for our calculation and will be needed for more general calculations of CMB bispectra.

\section{TWO-POINT CORRELATION FUNCTION AND POWER SPECTRUM}

We start by reviewing the calculation of the angular twopoint correlation function and power spectrum of CMB temperature fluctuations. The photon temperature perturbation at a spacetime point $(\mathbf{x}, \tau)$ can be Fourier expanded:

$$
\frac{\Delta T}{T}(\mathbf{x}, \tau, \hat{\mathbf{n}})=\int d^{3} k e^{i \mathbf{k} \cdot \mathbf{x}} \Delta(\mathbf{k}, \hat{\mathbf{n}}, \tau),
$$

where $\hat{\mathbf{n}}$ is the direction of photon momentum. We always set the observation point to be at the origin $(\mathbf{x}=0)$ and at the present epoch $\left(\tau=\tau_{0}\right)$, so we will not explicitly write these two variables in the following derivations. By Legendre expansion, we have

$$
\begin{aligned}
\frac{\Delta T}{T}(\hat{\mathbf{n}}) & =\int d^{3} k \Delta(\mathbf{k}, \hat{\mathbf{n}}) \\
& =\int d^{3} k \sum_{l=0}^{\infty}(-i)^{l}(2 l+1) \psi(\mathbf{k}) \Delta_{l}(k) P_{l}(\hat{\mathbf{k}} \cdot \hat{\mathbf{n}}),
\end{aligned}
$$

where $\psi(\mathbf{k})$ is the initial gravitational-potential perturbation, $\Delta_{l}(k)$ are photon transfer functions, and $P_{l}(\hat{\mathbf{k}} \cdot \hat{\mathbf{n}})$ are Legendre polynomials. We have used the fact that the photon evolution equation is independent of the wave vector direction $\hat{\mathbf{k}}$. For a stationary random process, we have

$$
\left\langle\psi\left(\mathbf{k}_{\mathbf{1}}\right) \psi\left(\mathbf{k}_{\mathbf{2}}\right)\right\rangle=P_{\psi}^{(2)}(k) \delta_{D}\left(\mathbf{k}_{1}+\mathbf{k}_{2}\right),
$$

where the amplitude $P_{\psi}^{(2)}(k)$ is the primordial power spectrum. For a scale-free primordial power spectrum, we have 


$$
P_{\psi}^{(2)}(k) \propto k^{n-4},
$$

where the index $n=1$ corresponds to a flat scale-invariant spectrum, which is close to those favored by generic inflationary models. The CMB temperature pattern may be written in a spherical-harmonic expansion with coefficients,

$$
a_{l m}=\int d \hat{\mathbf{n}} Y_{l m}(\hat{\mathbf{n}}) \frac{\Delta T}{T}(\hat{\mathbf{n}}),
$$

where $Y_{l m}(\hat{\mathbf{n}})$ are spherical harmonics. The angular twopoint correlation function is

$$
\xi\left(\hat{\mathbf{n}}_{1}, \hat{\mathbf{n}}_{2}\right) \equiv\left\langle\frac{\Delta T}{T}\left(\hat{\mathbf{n}}_{1}\right) \frac{\Delta T}{T}\left(\hat{\mathbf{n}}_{2}\right)\right\rangle=\sum_{m} \frac{2 l+1}{4 \pi} C_{l} P_{l}\left(\hat{\mathbf{n}}_{1} \cdot \hat{\mathbf{n}}_{2}\right),
$$

where

$$
C_{l}=(4 \pi)^{2} \int k^{2} d k P_{\psi}^{(2)}(k)\left|\Delta_{l}(k)\right|^{2}
$$

is the CMB power spectrum. We have used Eqs. (2) and (3) to obtain Eq. (6), and the spherical-harmonic addition theorem, orthonormality of spherical harmonics, and

$$
\left\langle a_{l_{1} m_{1}} a_{l_{2} m_{2}}^{*}\right\rangle=\delta_{l_{1} l_{2}} \delta_{m_{1} m_{2}} C_{l},
$$

to obtain Eq. (7).

\section{THREE-POINT CORRELATION FUNCTION AND BISPECTRUM}

If the primordial random density fluctuation is nonGaussian, then the three-point correlation function is in general non-vanishing. We then have

$$
\left\langle\psi\left(\mathbf{k}_{1}\right) \psi\left(\mathbf{k}_{2}\right) \psi\left(\mathbf{k}_{3}\right)\right\rangle=P_{\psi}^{(3)}\left(k_{1}, k_{2}, k_{3}\right) \delta_{D}\left(\mathbf{k}_{1}+\mathbf{k}_{2}+\mathbf{k}_{3}\right),
$$

where $P_{\psi}^{(3)}\left(k_{1}, k_{2}, k_{3}\right)$ is the spatial bispectrum of the gravitational potential. The angular three-point correlation function for the CMB can be written as

$$
\begin{aligned}
\xi\left(\hat{\mathbf{n}}_{1}, \hat{\mathbf{n}}_{2}, \hat{\mathbf{n}}_{3}\right) \equiv & \left\langle\frac{\Delta T}{T}\left(\hat{\mathbf{n}}_{1}\right) \frac{\Delta T}{T}\left(\hat{\mathbf{n}}_{2}\right) \frac{\Delta T}{T}\left(\hat{\mathbf{n}}_{3}\right)\right\rangle \\
= & \sum_{l_{i}, m_{i}}\left\langle a_{l_{1} m_{1}} a_{l_{2} m_{2}} a_{l_{3} m_{3}}\right\rangle Y_{l_{1} m_{1}}\left(\hat{\mathbf{n}}_{1}\right) \\
& \times Y_{l_{2} m_{2}}\left(\hat{\mathbf{n}}_{2}\right) Y_{l_{3} m_{3}}\left(\hat{\mathbf{n}}_{3}\right),
\end{aligned}
$$

where

$$
\begin{aligned}
& \left\langle a_{l_{1} m_{1}} a_{l_{2} m_{2}} a_{l_{3} m_{3}}\right\rangle=(4 \pi)^{3}(-i)^{l_{1}+l_{2}+l_{3}} \int d^{3} k_{1} d^{3} k_{2} d^{3} k_{3} \\
& \times Y_{l_{1} m_{1}}^{*}\left(\hat{\mathbf{k}}_{1}\right) Y_{l_{2} m_{2}}^{*}\left(\hat{\mathbf{k}}_{2}\right) Y_{l_{3} m_{3}}^{*}\left(\hat{\mathbf{k}}_{3}\right) \\
& \times \delta_{D}\left(\mathbf{k}_{1}+\mathbf{k}_{2}+\mathbf{k}_{3}\right) P_{\psi}^{(3)}\left(k_{1}, k_{2}, k_{3}\right) \\
& \times \Delta_{l_{1}}\left(k_{1}\right) \Delta_{l_{2}}\left(k_{2}\right) \Delta_{l_{3}}\left(k_{3}\right) \text {. }
\end{aligned}
$$

This last equation is obtained by using Eqs. (5),(2), and the spherical-harmonic addition theorem. By using

$$
\begin{gathered}
\delta_{D}\left(\mathbf{k}_{1}+\mathbf{k}_{2}+\mathbf{k}_{3}\right)=\frac{1}{(2 \pi)^{3}} \int_{-\infty}^{\infty} e^{i\left(\mathbf{k}_{1}+\mathbf{k}_{2}+\mathbf{k}_{3}\right) \cdot \mathbf{x}} d^{3} x, \\
e^{i \mathbf{k} \cdot \mathbf{x}}=4 \pi \sum_{l} i^{l} j_{l}(k x) \sum_{m} Y_{l m}(\hat{\mathbf{k}}) Y_{l m}^{*}(\hat{\mathbf{x}}),
\end{gathered}
$$

and the Gaunt integral

$$
\begin{aligned}
\int d & \Omega Y_{l_{1} m_{1}} Y_{l_{2} m_{2}} Y_{l_{3} m_{3}} \\
& =\sqrt{\frac{\left(2 l_{1}+1\right)\left(2 l_{2}+1\right)\left(2 l_{3}+1\right)}{4 \pi}} \\
& \times\left(\begin{array}{ccc}
l_{1} & l_{2} & l_{3} \\
0 & 0 & 0
\end{array}\right)\left(\begin{array}{ccc}
l_{1} & l_{2} & l_{3} \\
m_{1} & m_{2} & m_{3}
\end{array}\right),
\end{aligned}
$$

where $(\cdots)$ is the Wigner $3 j$ symbol, we obtain

$$
\left\langle a_{l_{1} m_{1}} a_{l_{2} m_{2}} a_{l_{3} m_{3}}\right\rangle=\left(\begin{array}{ccc}
l_{1} & l_{2} & l_{3} \\
m_{1} & m_{2} & m_{3}
\end{array}\right) B_{l_{1} l_{2} l_{3}},
$$

where

$$
\begin{aligned}
B_{l_{1} l_{2} l_{3}}= & (8 \pi)^{3} \sqrt{\frac{\left(2 l_{1}+1\right)\left(2 l_{2}+1\right)\left(2 l_{3}+1\right)}{4 \pi}}\left(\begin{array}{ccc}
l_{1} & l_{2} & l_{3} \\
0 & 0 & 0
\end{array}\right) \\
& \times \int k_{1}^{2} d k_{1} k_{2}^{2} d k_{2} k_{3}^{2} d k_{3} J_{l_{1} l_{2} l_{3}}\left(k_{1}, k_{2}, k_{3}\right) \\
& \times P_{\psi}^{(3)}\left(k_{1}, k_{2}, k_{3}\right) \Delta_{l_{1}}\left(k_{1}\right) \Delta_{l_{2}}\left(k_{2}\right) \Delta_{l_{3}}\left(k_{3}\right)
\end{aligned}
$$

is the CMB bispectrum. The integral

$$
J_{l_{1} l_{2} l_{3}}\left(k_{1}, k_{2}, k_{3}\right)=\int j_{l_{1}}\left(k_{1} x\right) j_{l_{2}}\left(k_{2} x\right) j_{l_{3}}\left(k_{3} x\right) x^{2} d x
$$

can be calculated relatively quickly by using the recurrence relations discussed in the Appendix. $j_{l}(k x)$ are spherical Bessel functions. Equation (16) provides a general formalism for calculating the bispectrum of the CMB starting from any primordial spatial bispectrum.

FMG defined a statistic $\hat{B}_{l}$ which is related to the bispectrum by

$$
\hat{B}_{l}=\frac{1}{(2 l+1)^{3 / 2}}\left(\begin{array}{ccc}
l & l & l \\
0 & 0 & 0
\end{array}\right)^{-1} B_{l l l},
$$

and this is especially easy to calculate from Eq. (16). Because of the symmetry, we can assume $k_{1}<k_{2}<k_{3}$ and introduce three parameters, $r, u$, and $v$, defined by 


$$
\begin{aligned}
& k_{1}=r, \\
& k_{2}=u r, \\
& k_{3}=(u+v) r,
\end{aligned}
$$

where $u>1$ and $0<v<1$, to ensure the triangle relation between the three $k$ 's. Finally, we get

$$
\begin{aligned}
\hat{B}_{l}= & 6 \frac{(8 \pi)^{3}}{\sqrt{4 \pi}} \int_{0}^{\infty} r^{8} \Delta_{l}(r) d r \int_{1}^{\infty} u^{2} \Delta_{l}(u r) d u \\
& \times \int_{0}^{1}(u+v)^{2} d v J_{l l l}(r, u r, r(u+v)) \\
& \times P_{\psi}^{(3)}(r, u r, r(u+v)) \Delta_{l}(r(u+v)) .
\end{aligned}
$$

The factor of 6 comes from permutation of $k_{1}, k_{2}$, and $k_{3}$.

At the large angular scales relevant for COBE, the SachsWolfe effect [24] dominates, and we simply have $\Delta_{l}(k)$ $=A_{\mathrm{sw}} j_{l}(k \Delta \eta)$, where $\Delta \eta=\left(\eta_{0}-\eta_{*}\right)$ is the conformal time between now and the surface of last scatter, and $A_{\mathrm{sw}}=1 / 3$ for a critical-density model with primordial adiabatic perturbations.

\section{SINGLE-FIELD SLOW-ROLL INFLATION}

The formalism outlined above is general and applicable to any form of $P_{\psi}^{(3)}$. However, as pointed out by Luo [25], the $P_{\psi}^{(3)}$ generated by slow-roll inflation models [12] has a special form that simplifies the calculation. In fact, the powerspectrum normalized bispectrum,

$$
I_{l}^{3} \equiv \frac{\hat{\mathbf{B}}_{l}}{\left(C_{l}\right)^{3 / 2}}
$$

which was extracted from the COBE Differential Microwave Radiometer (DMR) data by FMG, can be expressed in a very simple form for models with the following form of the spatial three-point correlation function:

$$
P_{\psi}^{(3)}\left(k_{1}, k_{2}, k_{3}\right)=f\left(k_{1}, k_{2}\right)+f\left(k_{2}, k_{3}\right)+f\left(k_{1}, k_{3}\right),
$$

where $f(x, y)$ is an arbitrary function of $x$ and $y$. We start with the Sachs-Wolfe formula

$$
\frac{\Delta T}{T}(\hat{\mathbf{n}})=A_{\mathrm{sw}} \int d^{3} k \psi(\mathbf{k}) e^{i \Delta \eta \mathbf{k} \cdot \hat{\mathbf{n}}}
$$

In this case, the angular three-point correlation function becomes

$$
\begin{aligned}
\xi\left(\hat{\mathbf{n}}_{1}, \hat{\mathbf{n}}_{2}, \hat{\mathbf{n}}_{3}\right)= & A_{\mathrm{sw}}^{3} \int d^{3} k_{1} d^{3} k_{2} d^{3} k_{3} e^{i \Delta \eta\left(\mathbf{k}_{1} \cdot \hat{\mathbf{n}}_{1}+\mathbf{k}_{2} \cdot \hat{\mathbf{n}}_{2}+\mathbf{k}_{3} \cdot \hat{\mathbf{n}}_{3}\right)} \\
& \times \delta_{D}\left(\mathbf{k}_{1}+\mathbf{k}_{2}+\mathbf{k}_{3}\right)\left[f\left(k_{1}, k_{2}\right)+f\left(k_{2}, k_{3}\right)\right. \\
& \left.+f\left(k_{1}, k_{3}\right)\right] .
\end{aligned}
$$

Now, let us calculate the first term associated with $f\left(k_{1}, k_{2}\right)$. By integrating out $k_{3}$, then using spherical-harmonic orthonormality, Eqs. (13) and (14), and

$$
\sum_{l m} Y_{l m}\left(\hat{\mathbf{n}}_{1}\right) Y_{l m}^{*}\left(\hat{\mathbf{n}}_{2}\right)=\delta_{D}\left(\hat{\mathbf{n}}_{1}-\hat{\mathbf{n}}_{2}\right)
$$

we have the bispectrum from the first term of Eq. (26),

$$
\begin{aligned}
& B_{l_{1} l_{2} l_{3}}(\text { first term }) \\
& =A_{\mathrm{sw}}^{3}(4 \pi)^{4} \sqrt{\frac{\left(2 l_{1}+1\right)\left(2 l_{2}+1\right)\left(2 l_{3}+1\right)}{4 \pi}} \\
& \quad \times\left(\begin{array}{ccc}
l_{1} & l_{2} & l_{3} \\
0 & 0 & 0
\end{array}\right) \\
& \quad \times \int d k_{1} d k_{2} k_{1}^{2} k_{2}^{2} f\left(k_{1}, k_{2}\right) j_{l_{1}}^{2}\left(k_{1} \Delta \eta\right) j_{l_{2}}^{2}\left(k_{2} \Delta \eta\right),
\end{aligned}
$$

and similarly for the second and third terms. The quantity $I_{l}^{3}$ then takes the form

$$
I_{l}^{3}=\sqrt{4 \pi} \frac{3 \int d k_{1} d k_{2} k_{1}^{2} k_{2}^{2} f\left(k_{1}, k_{2}\right) j_{l}^{2}\left(k_{1} \Delta \eta\right) j_{l}^{2}\left(k_{2} \Delta \eta\right)}{\left[\int d k k^{2} P_{\psi}^{(2)}(k) j_{l}^{2}(k \Delta \eta)\right]^{3 / 2}} .
$$

For slow-roll-inflation models, $f\left(k_{1}, k_{2}\right)$ takes the form $[12,13]$

$$
f\left(k_{1}, k_{2}\right)=A_{\text {infl }} P_{\psi}^{(2)}\left(k_{1}\right) P_{\psi}^{(2)}\left(k_{2}\right),
$$

where

$$
A_{\text {infl }}=\frac{25 m_{\mathrm{Pl}}^{2}}{48 \pi}\left[\left(\frac{V^{\prime}}{V}\right)^{2}-\frac{4 V^{\prime \prime}}{5 V}\right]
$$

is a constant depending on the height $V$ of the inflaton potential and its first and second derivatives, $V^{\prime}$ and $V^{\prime \prime}$, respectively, with respect to the inflaton $\phi[13]$. Thus,

$$
I_{l}^{3}=3 \sqrt{4 \pi} A_{\text {infl }}\left[\int d k k^{2} P_{\psi}^{(2)}(k) j_{l}^{2}(k \Delta \eta)\right]^{1 / 2} .
$$

For a flat scale-invariant spectrum of primordial density perturbations, $P_{\psi}^{(2)}(k)=A_{H} k^{-3}$, where (using our Fourier and scale-factor conventions)

$$
A_{H}=\frac{96 V^{3}}{25\left(V^{\prime}\right)^{2} m_{\mathrm{Pl}}^{6}} \text {. }
$$

We thus find that in terms of the usual slow-roll parameters [9],

$$
\epsilon \equiv \frac{m_{\mathrm{Pl}}^{2}}{16 \pi}\left(\frac{V^{\prime}}{V}\right)^{2}, \quad \eta \equiv \frac{m_{\mathrm{Pl}}^{2}}{8 \pi}\left[\frac{V^{\prime \prime}}{V}-\frac{1}{2}\left(\frac{V^{\prime}}{V}\right)^{2}\right],
$$


our central result for $I_{l}^{3}$ can be expressed as

$$
\sqrt{l(l+1)} I_{l}^{3}=\frac{2}{m_{\mathrm{Pl}}^{2}} \sqrt{\frac{3 V}{\epsilon}}(3 \epsilon-2 \eta) .
$$

Given that $\left(V^{1 / 2} / m_{\mathrm{PI}}^{2}\right) / \sqrt{\epsilon} \sim H^{2} / \dot{\phi} \sim 10^{-5}$ is the densityperturbation amplitude, and $\epsilon, \eta \leq 1$ in slow-roll inflation, it is clear that a value $\sqrt{l(l+1)} I_{l}^{3} \sim 1$ is inconsistent with single-field slow-roll inflation.

\section{SINGLE-FIELD MODEL WITH A FEATURE}

Equations (34) and (35) suggest that the bispectrum could be larger if the inflaton potential was not as smooth as required for slow-roll inflation. Thus, consider now an inflation model with a "feature" in the inflaton potential, such as in those models introduced to account for the claimed detection of a feature in the measured mass power spectrum $[15,19,26]$. More precisely, consider a single-field inflation model in which the inflaton potential is continuous, but its scaled slope $\delta \equiv m_{\mathrm{Pl}}\left(V^{\prime} / V\right)=4(\pi \epsilon)^{1 / 2}$ is discontinuous at $k_{0}$. The derivative of the scaled slope (with respect to the field $\phi)$ is proportional to a Dirac delta function, $\delta^{\prime}$ $=A_{\text {feat }} k_{0} m_{\mathrm{Pl}}^{-1} \delta_{D}\left(k-k_{0}\right)$. The spike occurs at the value that $\phi$ has when the comoving wave number $k_{0}$ exits the horizon, and $A_{\text {feat }}$ is the dimensionless amplitude of the delta function.

We must have $\delta \leqq 1$ on both sides of the delta function, and this places a constraint on the amplitude $A_{\text {feat }}$. The change to $\delta$ as the field passes through the Dirac delta function is

$$
\Delta \delta=\int \delta^{\prime} d \phi=\int \frac{A_{\text {feat }} k_{0}}{m_{\mathrm{Pl}}} \delta\left(k-k_{0}\right) d \phi,
$$

and this must be small compared with unity. Using $d \phi$ $=(d \phi / d \ln k) d \ln k \simeq(\dot{\phi} / H)(d k / k)$ and $\dot{\phi} / H \simeq\left(m_{\mathrm{Pl}}^{2} / 8 \pi\right)\left(V^{\prime} / V\right)$ during slow roll, we get $\Delta \delta \simeq A_{\text {feat }} \delta / 8 \pi$, and so $A_{\text {feat }} \leqslant 8 \pi$.

To proceed more precisely, we must realize that the derivation $[12,13]$ that leads to our Eq. (30) is valid only if the inflaton potential varies smoothly. By including the Dirac delta function in Eq. (32) in Ref. [13], we arrive at the correct expression for the spatial gravitational-potential bispectrum for an inflaton potential with a feature:

$$
\begin{aligned}
P_{\psi}^{(3)}\left(k_{1}, k_{2}, k_{3}\right)= & \frac{5}{6(2 \pi)^{4}} A_{\text {feat }} k_{0} \delta\left(k_{1}-k_{0}\right) P_{\psi}^{(2)}\left(k_{2}\right) P_{\psi}^{(2)}\left(k_{3}\right) \\
& +\left\{k_{1} \leftrightarrow k_{2}\right\}+\left\{k_{1} \leftrightarrow k_{3}\right\} .
\end{aligned}
$$

We can still approximate $P_{\psi}^{(2)}=A_{H} k^{-3}$ (since we are still considering only $\epsilon, \eta \ll 1$ other than the point $k=k_{0}$ ), and then from Eqs. (16) and (18), we have

$$
\begin{aligned}
\hat{\mathbf{B}}_{l}= & \frac{40}{3 \pi^{3 / 2}} A_{\text {feat }} A_{H}^{2} \int k_{1}^{2} d k_{1} k_{2}^{2} d k_{2} k_{3}^{2} d k_{3} J_{l l l}\left(k_{1}, k_{2}, k_{3}\right) \\
& \times \Delta_{l}\left(k_{1}\right) \Delta_{l}\left(k_{2}\right) \Delta_{l}\left(k_{3}\right) \frac{k_{0} \delta\left(k_{1}-k_{0}\right)}{k_{2}^{3} k_{3}^{3}} \\
& +\left\{k_{1} \leftrightarrow k_{2}\right\}+\left\{k_{1} \leftrightarrow k_{3}\right\} .
\end{aligned}
$$

By the symmetry of $k_{1}, k_{2}$, and $k_{3}$, we obtain

$$
\begin{aligned}
I_{l}^{3}= & \left(\frac{H}{m_{\mathrm{Pl}} \delta}\right) \frac{6}{\pi^{2}} A_{\text {feat }} \widetilde{k}_{0}^{3} \Delta_{l}\left(\widetilde{k}_{0}\right) \\
& \times\left(\int_{0}^{\infty}\left|\Delta_{l}(\widetilde{k})\right|^{2} \widetilde{k}^{-1} d \widetilde{k}\right)^{-3 / 2} \\
& \times \int_{0}^{\infty} \Delta_{l}\left(\widetilde{k}_{2}\right) \widetilde{k}_{2}^{-1} d \widetilde{k}_{2} \int_{\left|\widetilde{k}_{0}-\widetilde{k}_{2}\right|}^{\tilde{k}_{0}+\widetilde{k}_{2}} \Delta_{l}\left(\widetilde{k}_{3}\right) \\
& \times j_{l l l}\left(\widetilde{k}_{0}, \widetilde{k}_{2}, \widetilde{k}_{3}\right) \widetilde{k}_{3}^{-1} d \widetilde{k}_{3},
\end{aligned}
$$

where $\widetilde{k}_{x}=k_{x} \Delta \eta$. Again, during slow roll, the first term $H /\left(m_{\mathrm{P} \mid} \delta\right) \sim H^{2} / \dot{\phi} \sim 10^{-5}$. Numerical integration shows that the rest expression peaks at a value of $l$ corresponding to the scale of $k_{0}$, but with an amplitude $\lesssim 0.1 A_{\text {feat }}$. Therefore, even with a discontinuity in the slope of the inflaton potential, the predicted $I_{l}^{3}$ is still several orders of magnitude smaller than unity (the desired value to explain the COBE anomaly found by FMG around $l=16$ ) as long as the slowroll conditions are satisfied everywhere else.

\section{DISCUSSION}

We gave a numerically manageable general formalism that allows us to calculate the bispectrum of the CMB starting from an arbitrary spatial bispectrum. For certain forms of the spatial bispectrum, including those given by single-field slow-roll inflation models, the Sachs-Wolfe calculation results in an analytic expression for the non-Gaussian statistic $I_{l}^{3}$; it turns out to be roughly the density-perturbation amplitude times a linear combination of the slow-roll parameters $\epsilon$ and $\eta$. This result demonstrates that FMG's non-Gaussian signal is inconsistent with single-field slow-roll inflation models. The predicted value of $I_{l}^{3}$ can be increased if the slope of the inflaton potential is discontinuous, but even this larger non-Gaussian signal is too small to account for the detection found in Ref. [1]. Although a complete calculation would require detailed specification of a model, our results for the discontinuous single-field model suggest that generic multiple-field models designed to produce a break in the slope of the mass power spectrum $[18,19]$ will be unable to produce a non-Gaussian signal large enough to explain the COBE result. Counter-examples can be found. In particular, inflation models can be designed in which density perturbations are produced by quantum fluctuations of some scalar field other than that driving inflation. For example, in Ref. [20], non-Gaussian isocurvature perturbations to an axion density are produced by quantum fluctuations in the axion 
field, but inflation is driven by some other scalar field not associated with Peccei-Quinn symmetry breaking. The mechanism of Refs. [22,23] is somewhat similar. In the model of Ref. [21], non-Gaussian density perturbations are produced (at least in part) by the square of a second scalar field (although details of the inflationary dynamics are not presented). In conclusion, if the detection of nonzero $I_{l}^{3}$ is ultimately attributed to the $\mathrm{CMB}$, it raises a serious problem for inflationary models where quantum fluctuations in the inflaton give rise to large-scale structure.

Note added. After we posted our paper, we noticed a con- current work by Gangui and Martin [27] that is closely related to the subject we discussed here.

\section{ACKNOWLEDGMENTS}

We thank R. Friedberg for suggesting the recursive technique for evaluating integrals with oscillatory integrands. We thank K. Górski, A. Kosowsky, J. Lesgourges, J. Magueijo, and L. Verde for useful comments and discussions. This work was supported by the DOE Outstanding Junior Investigator program, DE-FG02-92ER40699, NASA grant NAG53091, and the Alfred P. Sloan Foundation.

\section{APPENDIX}

We need to evaluate the following integral:

$$
J_{l_{1} l_{2} l_{3}}\left(k_{1}, k_{2}, k_{3}\right)=\int_{0}^{\infty} j_{l_{1}}\left(k_{1} x\right) j_{l_{2}}\left(k_{2} x\right) j_{l_{3}}\left(k_{3} x\right) x^{2} d x,
$$

where $j_{l}(x)$ are spherical Bessel functions. The wave numbers $k_{1}, k_{2}$, and $k_{3}$ satisfy the triangle relation (they should be able to form a triangle), and $l_{1}+l_{2}+l_{3}$ has to be an even number. To evaluate the integral, we use the recursive technique discussed in Ref. [28]. To begin, we evaluate the integral for some special cases:

$$
\begin{aligned}
& J_{l 00}=\int_{0}^{\infty} \frac{1}{2 i^{l}} \int_{-1}^{1} P_{l}(u) e^{i u k_{1} x} d u \frac{\sin k_{2} x}{k_{2} x} \frac{\sin k_{3} x}{k_{3} x} x^{2} d x \\
& \stackrel{l \text { even }}{=} \frac{1}{2 i^{l}} \frac{1}{k_{2} k_{3}} \int_{-1}^{1} P_{l}(u) d u \int_{-\infty}^{\infty} e^{i u k_{1} x} \sin k_{2} x \sin k_{3} x d x \\
& =\frac{-\pi}{4 i^{l} k_{2} k_{3}} \int_{0}^{1} P_{l}(u) d u\left[\delta\left(u k_{1}+k_{2}+k_{3}\right)-\delta\left(u k_{1}-k_{2}+k_{3}\right)-\delta\left(u k_{1}+k_{2}-k_{3}\right)+\delta\left(u k_{1}-k_{2}-k_{3}\right)\right] \\
& \stackrel{\Delta \text { relation }}{=} \frac{\pi}{4 i^{l} k_{2} k_{3}} \int_{0}^{1} P_{l}(u) d u \delta\left(u k_{1}-\left|k_{2}-k_{3}\right|\right)=\frac{\pi}{4 i^{l}} \frac{1}{k_{1} k_{2} k_{3}} P_{l}\left(\frac{k_{2}-k_{3}}{k_{1}}\right), \\
& J_{l,-1,0}=\int_{0}^{\infty} \frac{1}{2 i^{l}} \int_{-1}^{1} P_{l}(u) e^{i u k_{1} x} d u \frac{\cos k_{2} x}{k_{2} x} \frac{\sin k_{3} x}{k_{3} x} x^{2} d x=\frac{1 \text { odd }}{2 i^{l}} \frac{1}{k_{2} k_{3}} \int_{-1}^{1} P_{l}(u) d u \int_{-\infty}^{\infty} e^{i u k_{1} x} \cos k_{2} x \sin k_{3} x d x \\
& =\frac{\pi}{4 i^{l+1} k_{2} k_{3}} \int_{0}^{1} P_{l}(u) d u\left[\delta\left(u k_{1}+k_{2}+k_{3}\right)+\delta\left(u k_{1}-k_{2}+k_{3}\right)-\delta\left(u k_{1}+k_{2}-k_{3}\right)-\delta\left(u k_{1}-k_{2}-k_{3}\right)\right] \\
& \stackrel{\Delta \text { relation }}{=} \frac{\pi}{4 i^{l+1} k_{2} k_{3}} \int_{0}^{1} P_{l}(u) d u\left[\delta\left(u k_{1}-k_{2}+k_{3}\right)-\delta\left(u k_{1}+k_{2}-k_{3}\right)\right] \\
& =\frac{\pi}{4 i^{l+1}} \frac{1}{k_{1} k_{2} k_{3}} P_{l}\left(\frac{k_{2}-k_{3}}{k_{1}}\right), \\
& J_{l,-1,-1}=\frac{\pi}{4 i^{l}} \frac{1}{k_{1} k_{2} k_{3}} P_{l}\left(\frac{k_{2}-k_{3}}{k_{1}}\right) .
\end{aligned}
$$

To evaluate the integral for other cases, we use the recursion relation,

$$
j_{l-1}(\alpha)+j_{l+1}(\alpha)=\frac{2 l+1}{\alpha} j_{l}(\alpha),
$$

to obtain 


$$
\begin{aligned}
D_{l_{1} l_{2} l_{3}}\left(k_{1}, k_{2}, k_{3}\right) & =\int_{0}^{\infty} j_{l_{1}}\left(k_{1} x\right) j_{l_{2}}\left(k_{2} x\right) j_{l_{3}}\left(k_{3} x\right) x d x=\frac{k_{1}}{2 l_{1}+1}\left(J_{l_{1}-1, l_{2}, l_{3}}+J_{l_{1}+1, l_{2}, l_{3}}\right) \\
& =\frac{k_{2}}{2 l_{2}+1}\left(J_{l_{1}, l_{2}-1, l_{3}}+J_{l_{1}, l_{2}+1, l_{3}}\right) .
\end{aligned}
$$

Therefore, we have the recursion relation we need:

$$
J_{l_{1}, l_{2}+1, l_{3}}=\frac{k_{1}}{k_{2}} \frac{2 l_{2}+1}{2 l_{1}+1}\left(J_{l_{1}-1, l_{2}, l_{3}}+J_{l_{1}+1, l_{2}, l_{3}}\right)-J_{l_{1}, l_{2}-1, l_{3}} .
$$

If we begin with Eqs. (A2), (A3), and (A4), then we can use Eq. (A8), to recursively evaluate the integral (A1) for any combination of $l_{1}, l_{2}$, and $l_{3}$.

[1] P. G. Ferreira, J. Magueijo, and K. M. Górski, Astrophys. J. Lett. 503, L1 (1998).

[2] J. Pando, D. Valls-Gabaud, and L.-Z. Fang, Phys. Rev. Lett. 81, 4568 (1998).

[3] D. Novikov, H. Feldman, and S. Shandarin, Int. J. Mod. Phys. D 8, 29 (1999).

[4] A. F. Heavens, Mon. Not. R. Astron. Soc. 299, 805 (1998).

[5] M. Kamionkowski and A. H. Jaffe, Nature (London) 395, 639 (1998).

[6] B. C. Bromley and M. Tegmark, astro-ph/9904254.

[7] P. G. Ferreira, K. M. Górski, and J. Magueijo, astro-ph/9904073.

[8] A. J. Banday, S. Zaroubi, and K. M. Górski, astro-ph/9908070.

[9] M. Kamionkowski and A. Kosowsky, Annu. Rev. Nucl. Part. Sci. (to be published), astro-ph/9904108.

[10] D. S. Salopek, J. R. Bond, and J. M. Bardeen, Phys. Rev. D 40, 1753 (1989).

[11] D. S. Salopek, Phys. Rev. D 45, 1139 (1992).

[12] T. Falk, R. Rangarajan, and M. Srednicki, Astrophys. J. Lett. 403, L1 (1993).

[13] A. Gangui, F. Lucchin, S. Matarrese, and S. Mollerach, Astrophys. J. 430, 447 (1994).
[14] A. Gangui, Phys. Rev. D 50, 3684 (1994).

[15] J. Einasto et al., Nature (London) 385, 139 (1997).

[16] J. Peacock, Mon. Not. R. Astron. Soc. 284, 885 (1997).

[17] E. Gaztanaga and C. M. Baugh, Mon. Not. R. Astron. Soc. 294, 229 (1998).

[18] A. A. Starobinsky, JETP Lett. 55, 489 (1992).

[19] J. Lesgourgues, D. Polarski, and A. A. Starobinsky, Mon. Not. R. Astron. Soc. 297, 769 (1998).

[20] T. J. Allen, B. Grinstein, and M. Wise, Phys. Lett. B 197, 66 (1987).

[21] Z. H. Fan and J. M. Bardeen, KEK library scanned Report No. 9207442, UW-PT-92-11 (1992).

[22] A. Linde and V. Mukhanov, Phys. Rev. D 56, 535 (1997).

[23] P. J. E. Peebles, Astrophys. J. 510, 523 (1999); 510, 531 (1999).

[24] R. K. Sachs and A. M. Wolfe, Astrophys. J. 147, 73 (1967).

[25] X. Luo, Astrophys. J. Lett. 427, L71 (1994).

[26] I. Zlatev, L. Wang, R. R. Caldwell, and P. J. Steinhardt (in preparation).

[27] A. Gangui and J. Martin, astro-ph/9908009.

[28] R. Friedberg and O. Martin, J. Phys. A 20, 5095 (1987). 